\title{
5-Lipoxygenase-Activating Protein as a Modulator of Olanzapine-Induced Lipid Accumulation in Adipocyte
}

\author{
Svetlana Dzitoyeva, Hu Chen, and Hari Manev \\ Department of Psychiatry, Psychiatric Institute, University of Illinois at Chicago, 1601 West Taylor Street, \\ MC912, Chicago, IL 60612, USA
}

Correspondence should be addressed to Hari Manev; hmanev@psych.uic.edu

Received 12 March 2013; Revised 8 May 2013; Accepted 10 May 2013

Academic Editor: Akihiro Inazu

Copyright (C) 2013 Svetlana Dzitoyeva et al. This is an open access article distributed under the Creative Commons Attribution License, which permits unrestricted use, distribution, and reproduction in any medium, provided the original work is properly cited.

\begin{abstract}
Experiments were performed in 3T3-L1 preadipocytes differentiated in vitro into adipocytes. Cells were treated with olanzapine and a 5-lipoxygenase (5-LOX) activating protein (FLAP) inhibitor MK-886. Lipid content was measured using an Oil Red O assay; 5LOX and FLAP mRNA content was measured using quantitative real-time PCR; the corresponding protein contents were measured using quantitative Western blot assay. Olanzapine did not affect the cell content of 5-LOX mRNA and protein; it decreased FLAP mRNA and protein content at day five but not 24 hours after olanzapine addition. In the absence of MK-886, low concentrations of olanzapine increased lipid content only slightly, whereas a $56 \%$ increase was induced by $50 \mu \mathrm{M}$ olanzapine. A 5 -day cotreatment with $10 \mu \mathrm{M}$ MK-886 potentiated the lipid increasing action of low concentrations of olanzapine. In contrast, in the presence of $50 \mu \mathrm{M}$ olanzapine nanomolar and low micromolar concentrations of MK-886 reduced lipid content. These data suggest that FLAP system in adipocytes is affected by olanzapine and that it may modify how these cells respond to the second-generation antipsychotic drugs (SGADs). Clinical studies could evaluate whether the FLAP/5-LOX system could play a role in setting a variable individual susceptibility to the metabolic side effects of SGADs.
\end{abstract}

\section{Introduction}

Serious side effects hamper pharmacological treatment of psychiatric illnesses such as schizophrenia and bipolar disorder. All currently used second-generation antipsychotic drugs (SGADs) including olanzapine are capable of triggering significant weight gain associated with adverse metabolic alterations $[1,2]$. It has been proposed that these side effects are caused by a combination of factors including increased fat deposition [3] and appetite stimulation [4]. Interestingly, it was noted that SGADs are capable of increasing adiposity even in the absence of significant body weight gain [57] presumably by drug-impaired lipolysis [7, 8] and/or a direct stimulatory action of SGADs on adipocytes [9-11]. In the therapy of psychiatric patients with SGADs, a better understanding of the mechanisms that lead to this clinical problem is needed to identify the risk factors that facilitate and exacerbate these side effects of SGADs and to develop methods and therapies to prevent and/or treat their occurrence.

Recently, an in vitro model of 3T3-L1 cells has been developed to study the effects of olanzapine on peripheral adipogenesis [10]. Unrelated studies with this cell culture model have shown that the enzymatic pathway of 5-lipoxygenase (5LOX), which typically is involved in the association between adipose tissues and inflammation [12, 13], may contribute to lipid dysfunction [14]. Interestingly, the olanzapine-induced enlargement of adipose tissue also has been associated with inflammation [15].

5-LOX gene polymorphism and the associated 5-LOX enzyme deficiency occur generally in humans and have been noted as an example of pharmacogenetics [16]. In mice, a 5-LOX deficiency (e.g., gene knockdown) is accompanied by increased adiposity and elevated plasma levels of leptin, a factor produced by adipocytes that acts in the brain $[17,18]$. For its full activity, 5-LOX translocates to the nuclear 
membrane where it interacts with a transmembrane protein five lipoxygenase activating protein (FLAP) [19]. Both in human [20] and mouse [14] adipose tissue, FLAP expression is increased in obesity. Furthermore, a high-fat diet in mice caused a transient increase in the FLAP mRNA levels (after a 4-week treatment; returning to the basal levels at weeks 6 and 8) in aortic endothelium [21]. On the other hand, an acute fat load in healthy humans reduced FLAP expression in peripheral blood mononuclear cells [22]. Increased FLAP levels in adipose tissue are associated with increased levels of 5-LOX products, for example, leukotriene $\mathrm{LTB}_{4}$ that is capable of inducing the NF- $\kappa \mathrm{B}$ signaling pathway in adipocytes [14]. In obesity, a beneficial effect of pharmacological FLAP inhibition has been attributed to an induction of AMPactivated protein kinase phosphorylation and a concomitant decrease in hormone-sensitive lipase activity accompanied by reduced steatosis [14].

To our knowledge, the 5-LOX system has not been investigated in relation to the SGADs treatment. In this work, we used the 3T3-L1 cell culture model to investigate the putative association of the 5-LOX/FLAP system with olanzapine-triggered lipid accumulation. The preliminary results of this study have been reported in an abstract form [23].

\section{Material and Methods}

2.1. Cell Cultures and Drug Treatment. The 3T3-L1 preadipocytes (ATCC, American Type Culture Collection) were grown at $37^{\circ} \mathrm{C}, 5 \% \mathrm{CO}_{2}$ air, in $35 \times 10 \mathrm{~mm}$ tissue culture dishes (Falcon, Fisher) containing $2 \mathrm{~mL}$ of high glucose Dulbecco's modified Eagle's medium (DMEM, GIBCO) supplemented with $10 \%$ fetal bovine serum (FBS, Atlanta Biologicals). When the plated preadipocytes reached confluence, they were differentiated into adipocytes by changing the culture medium to DMEM/10\% FBS medium supplemented with $1 \mu \mathrm{g} / \mathrm{mL}$ insulin, $1 \mu \mathrm{M}$ dexamethasone, and $0.5 \mathrm{mM}$ 3-isobutyl-1-methylxanthine (IBMX) (all from Sigma). This was considered day 0 in vitro. Cells were maintained in vitro for 5 days changing their medium at days 2 and 4 with fresh DMEM/10\% FBS medium supplemented with $1 \mu \mathrm{g} / \mathrm{mL}$ of insulin. Penicillin/streptomycin solution (1:100; GIBCO, Grand Island, NY, USA) was added with every medium addition as a contamination preventive measure. The experimental drugs olanzapine (Sequoia Research Products Ltd., Pangbourne, UK) and MK-886 (Cayman Chemical, Ann Arbor, MI, USA) or their vehicle (1:1000 dimethyl sulfoxide, DMSO; Sigma) were added at day 0 and in the refreshment medium at days 2 and 4 (adapted from [10]).

2.2. Oil Red $O$ Assay. The culture medium was gently removed with a transfer pipette, and $1 \mathrm{~mL}$ of $12 \%$ formaldehyde in phosphate buffer saline was applied on top of the cells. After $20 \mathrm{~min}$, cells were rinsed three times with $2 \mathrm{~mL}$ of distilled water and covered with $1 \mathrm{~mL}$ of Oil Red O (Sigma) solution ( $0.5 \%$ Oil Red O in isopropanol diluted with water $3: 2$ and filtered through a $0.45 \mu \mathrm{m}$ filter) for $30 \mathrm{~min}$ at room temperature. The Oil Red $\mathrm{O}$ solution was discarded with a transfer pipette, and cells were rinsed three times with $2 \mathrm{~mL}$ of distilled water. After removing all water, Oil Red $\mathrm{O}$ dye captured by intracellular lipids was recovered with $400 \mu \mathrm{L}$ of isopropanol applied drop by drop on top of the cells. The resulting solution was transferred into microcentrifuge tubes and its absorbance values were measured in a microplate reader (Bio-Rad, Model 550) in a single wavelength mode with a $520 \mathrm{~nm}$ filter. For the visualization of the Oil Red O, staining cells were grown on glass cover slips. They were fixed and stained as described above. Microphotographs were taken after transferring the cover slips onto a glass slide and covered them with a mounting solution.

2.3. RNA Extraction and Quantitative Real-Time PCR. Total RNA was extracted with TRIzol reagent (Invitrogen Carlsbad, CA, USA) according to the manufacturer's instructions and treated with DNase (Ambion Inc., Austin, TX, USA). Thereafter, $1 \mu \mathrm{g}$ of RNA was reverse transcribed with M-MLV reverse transcriptase (Invitrogen, Carlsbad, CA, USA). Random hexamer primers and dNTPs were purchased from Fermentas (Fermentas Inc., Glen Burnie, MD, USA). The quantitative real-time PCR was performed on Stratagene Mx3005P QPCR System (Agilent Technologies) with a PCR SYBR Green Master mix (Fermentas Inc., Glen Burnie, MD, USA). Primers used: 5-LOX forward $5^{\prime}$-attgccatccagctcaaccaaacc$3^{\prime}$, reverse $5^{\prime}$-tggcgataccaaacacctcagaca- $3^{\prime}$; FLAP forward $5^{\prime}$ ccacaaggtggagcatgaaagcaa- $3^{\prime}$, reverse $5^{\prime}$-aaacaggtacatcagtccggcgaa- $3^{\prime}$; cyclophilin forward $5^{\prime}$-agcatacaggtcctggcatcttgt$3^{\prime}$, reverse $5^{\prime}$-aaacgctccatggcttccacaatg- $3^{\prime}$. Data were normalized against the corresponding cyclophilin internal control and presented as a coefficient of variation calculated with a formula $2^{-[\Delta C t(\text { target })-\Delta C(\text { input })]}[24]$.

2.4. Western Blot Assay. Cells were homogenized in $200 \mu \mathrm{L}$ ice cold RIPA buffer with protease inhibitors $(5 \mu \mathrm{g} / \mathrm{mL}$ aprotinin, $5 \mu \mathrm{g} / \mathrm{mL}$ leupeptin, $5 \mu \mathrm{g} / \mathrm{mL}$ pepstatin, $1 \mathrm{mM}$ phenylmethanesulfonylfluoride) and then centrifuged at $10000 \mathrm{~g}$ for $15 \mathrm{~min}$. The supernatant was stored at $-80^{\circ} \mathrm{C}$. After measuring protein concentration with a BCA protein assay kit (Pierce, Rockford, IL, USA), samples of $30 \mu \mathrm{g}$ proteins were boiled in loading buffer and run on $7.5 \%$ or $10 \%$ gels. Proteins were then transferred to nitrocellulose membranes. After blocking by $5 \%$ nonfat dry milk for $1 \mathrm{~h}$, the membranes were incubated overnight at $4^{\circ} \mathrm{C}$ with rabbit anti-FLAP antibody $(1: 500$, Santa Cruz, Santa Cruz, CA, USA), mouse anti-5-LOX antibody (1:1000, BD Bioscience, San Jose, CA, USA), or mouse anti- $\beta$-actin antibody (1:5000, Sigma, St. Louis, MO, USA). Thereafter, blots were incubated for $2 \mathrm{~h}$ with horseradishperoxidase-linked secondary antibodies. The membranes were developed with an ECL Kit (Amersham). Band densities were quantified using $\mathrm{NIH}$ Image software. The optical density of FLAP and 5-LOX bands was corrected by the corresponding $\beta$-actin bands.

2.5. Statistics. Statistical analyses were performed using SPSS software (SPSS Inc., Chicago, IL, USA). Data (shown as mean \pm SEM) were analyzed by one-way analysis of variance 
(ANOVA) followed by post hoc multiple comparison with Bonferroni correction or Student's $t$-test. Significance was accepted at $P<0.05$.

\section{Results}

To study the in vitro effects of olanzapine, we used a previously published protocol [10] for olanzapine-increased adipogenesis in 3T3-L1 cells. With this protocol, the preadipocytes, which expressed only a weak lipid staining (Figure 1(a)), in 5 days, differentiate into oil droplet-enriched adipocytes (Figure 1(b)). Addition of olanzapine to the culture medium of cells undergoing adipocyte differentiation resulted in an increase of lipid content in these cells (Figure 2). The difference in lipid content between olanzapine-treated and vehicletreated cultures was significant 24 hours after olanzapine addition and remained so at each day of in vitro treatment, that is, until day five (Figure 2). In this model, olanzapine treatment did not affect the cell content of 5-LOX mRNA; however, it decreased FLAP mRNA content at day five but not 24 hours after olanzapine addition to the culture medium (Figure 3). The inhibitory effect of olanzapine on FLAP expression was confirmed by quantitative Western blot assay. Hence, the content of FLAP protein at day five of olanzapine treatment was significantly lower compared to vehicle-treated controls. The content of 5-LOX protein was not affected by olanzapine (Figure 4). Addition of a high concentration of FLAP inhibitor MK-886 $(10 \mu \mathrm{M})$ during the 5 days of olanzapine treatment enhanced the adipogenic effect of olanzapine (Figure 5). Hence, in the absence of MK-886, low concentrations of olanzapine $(0.5$ and $5 \mu \mathrm{M})$ increased lipid content only by about 13\% (compared to about a $56 \%$ increase induced by $50 \mu \mathrm{M}$ olanzapine), whereas in the presence of $10 \mu \mathrm{M} \mathrm{MK}-886$, these concentrations of olanzapine produced lipid increases comparable to the increase caused by $50 \mu \mathrm{M}$ olanzapine (Figure 5). In contrast, low concentrations of MK-886 $(0.1-3 \mu \mathrm{M})$ reduced the lipid content of cells treated with the high $50 \mu \mathrm{M}$ concentration of olanzapine (Table 1).

\section{Discussion}

In this study, we show evidence that exposure of differentiating preadipocytes to olanzapine, in addition to increasing their accumulation of lipids, alters the expression of FLAP and that a FLAP inhibitor, MK-886, used in nanomolar and low micromolar concentrations decreases the adipogenic effects of a high concentration of olanzapine, whereas a $10 \mu \mathrm{M}$ concentration of MK-886 potentiated the adipogenic effect of low concentrations of olanzapine.

FLAP is an important regulatory protein of the 5LOX enzymatic pathway. In association with 5-LOX protein, FLAP regulates the synthesis of biologically active lipids, leukotrienes, and lipoxins $[19,25]$. Historically, this pathway has been studied for its role in inflammation [16] and also for its putative role in central nervous system disorders such as Alzheimer's disease [26-31] and obesity [12-14, 17, 18]. It has been suggested that obesity is accompanied by adipose
TABLE 1: Effect of low concentrations of MK-886 in the presence of $50 \mu \mathrm{M}$ olanzapine on intracellular lipid content.

\begin{tabular}{lc}
\hline Treatment (5 days) & Lipid content $^{\mathrm{a}}$ \\
\hline Olanzapine + Vehicle & $8.3 \pm 0.20$ \\
Olanzapine $+0.1 \mu \mathrm{M}$ MK-886 & $7.5 \pm 0.15^{*}$ \\
Olanzapine $+1.0 \mu \mathrm{M}$ MK-886 & $7.3 \pm 0.15^{* *}$ \\
Olanzapine $+3.0 \mu \mathrm{M}$ MK-886 & $7.2 \pm 0.08^{* *}$ \\
\hline
\end{tabular}

${ }^{a}$ Optical density readings at $520 \mathrm{~nm} \times 10($ mean \pm SEM; $n=3)$.

${ }^{*} P<0.05$.

${ }^{* *} P<0.01$

tissue dysfunction associated with a state of chronic mild inflammation evidenced by infiltration of inflammatory cells into adipose tissue $[13,32]$. In fact, studies on human and mouse obesity have demonstrated an increased presence of FLAP in the obese adipose tissue $[14,20]$, which was parallel with macrophage infiltration [14]. Furthermore, treatment with a 5-LOX inhibitor was shown to be capable of reducing the obesity-associated inflammation [12].

At the cellular level, multiple mechanisms including lipoxygenases are involved in differentiation of preadipocytes into adipocytes and in their intracellular lipid accumulation [33]. Using a previously established model of olanzapinetriggered adipogenesis in 3T3-L1 cells [10], we confirmed earlier findings that $50 \mu \mathrm{M}$ olanzapine (and to a lesser extent also $0.5-5.0 \mu \mathrm{M}$ olanzapine) significantly increases cellular lipid content. It was previously shown that this effect of olanzapine can be attributed to a drug-induced expression of an SREBP-1 regulatory protein (sterol regulatory element binding protein-1) [10]. SREBP-1 was also upregulated in 3T3L1 cells treated with another SGAD, clozapine [11]. In our experimental conditions, the adipogenic effect of olanzapine treatment was accompanied by a reduced expression of FLAP mRNA and protein, whereas the expression of 5-LOX was not significantly altered. The effect of olanzapine on FLAP content was not immediate; for example, it was not observed $24 \mathrm{~h}$ after the initiation of adipocyte differentiation, and it may have been set in motion by an initial lipid accumulation caused by an action of olanzapine on targets such as SREBP1. In fact, it has been reported that a fat overload per se can suppress FLAP expression [22].

Functional implications of FLAP protein in adipocytes have been suggested by the results of in vitro and in vivo experiments with a FLAP inhibitor Bay-X-1005 [14]. This drug not only reduced the inflammatory responses in adipocytes (e.g., it caused a downregulation of inflammatory markers such as tumor necrosis factor- $\alpha$ and interleukin6) but also in vivo Bay-X-1005 prevented hepatic steatosis created in a mouse model of dietary obesity [14]. In our experimental conditions, the nanomolar and low micromolar concentrations of MK-886 that are selective for FLAP inhibition reduced lipid content of cells treated with a high concentration of olanzapine. It was proposed that a FLAPmediated synthesis of 5-LOX products in adipocytes may lead to low-grade inflammation with lipid dysfunction and adiposity [14]. In addition, it was shown that pharmacological inhibition of lipoxygenases may interfere with adipocyte 


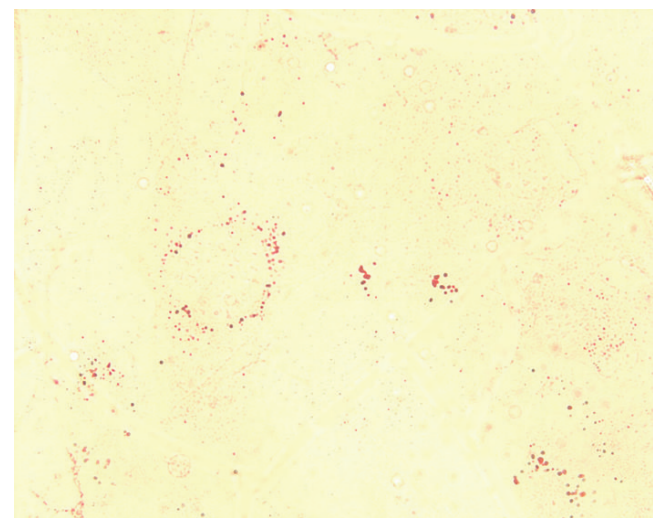

(a)

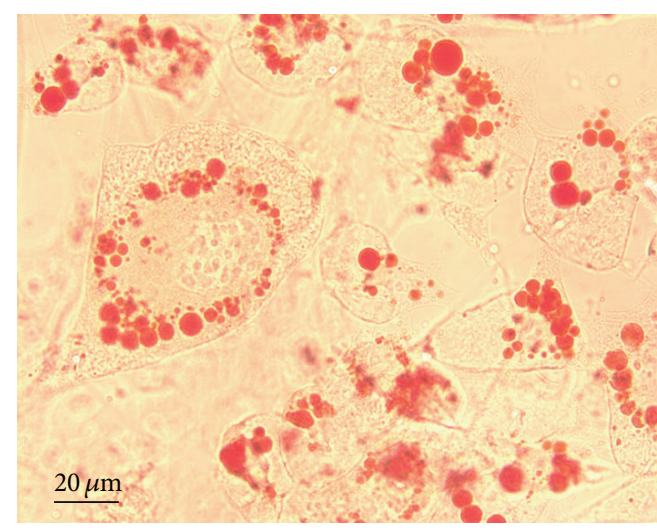

(b)

FIGURE 1: Oil Red O staining in undifferentiated 3T3-L1 cells (a) and after 5 days in vitro of adipocyte differentiation (b). Note the red/dark staining of oil droplets in B ( $\times 40$ objective lens).

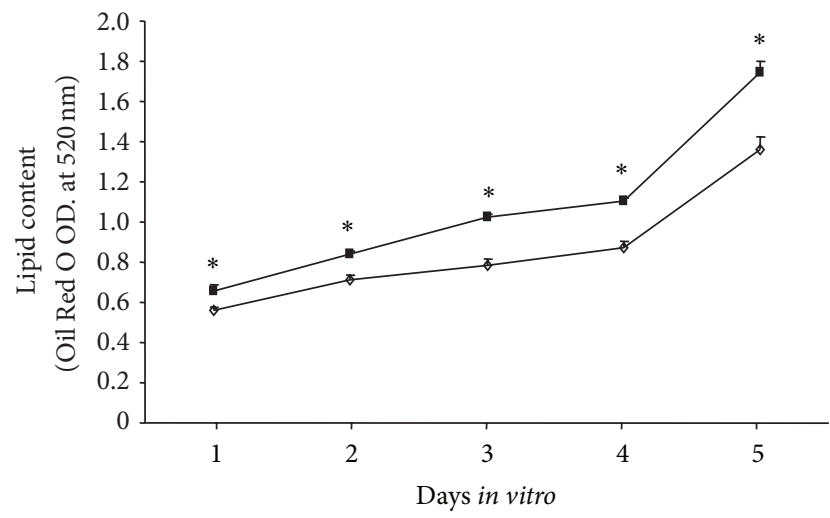

$\diamond$ Vehicle

- Olanzapine

FIGURE 2: Effect of olanzapine on intracellular lipid content measured by a quantitative Oil Red O assay. 3T3-L1 cells were differentiated into adipocytes in the presence and absence of $50 \mu \mathrm{M}$ olanzapine. Samples ( 3 culture dishes per treatment and time point) were collected daily starting 24 hours after the initiation of the differentiation protocol. Results (mean \pm SEM) are expressed as optical density (O.D.) readings at $520 \mathrm{~nm}$. At each time point, the values of olanzapine-treated cultures differed significantly from the values measured in corresponding vehicle-treated cultures $\left({ }^{*} \mathrm{P}<\right.$ 0.05 ; $t$-test).

differentiation and adipogenesis [3]. Our results suggest that a FLAP-dependent lipoxygenase pathway may be involved in the cellular actions of olanzapine on adipocytes.

On the other hand, we found that in contrast to FLAPspecific low concentrations of $\mathrm{MK}-886$, a higher $10 \mu \mathrm{M}$ concentration of MK-886 enhanced the adipogenic effect of a low (e.g., $0.5 \mu \mathrm{M}$ ) concentration of olanzapine. This action of MK-886 may be a FLAP-unrelated effect of the drug. Namely, it was shown that at $10 \mu \mathrm{M}$ MK-886 acts as a potent inhibitor of the peroxisome-proliferator-activated receptoralpha $(\operatorname{PPAR} \alpha)$ [34]. Hence, it is possible that PPAR $\alpha$

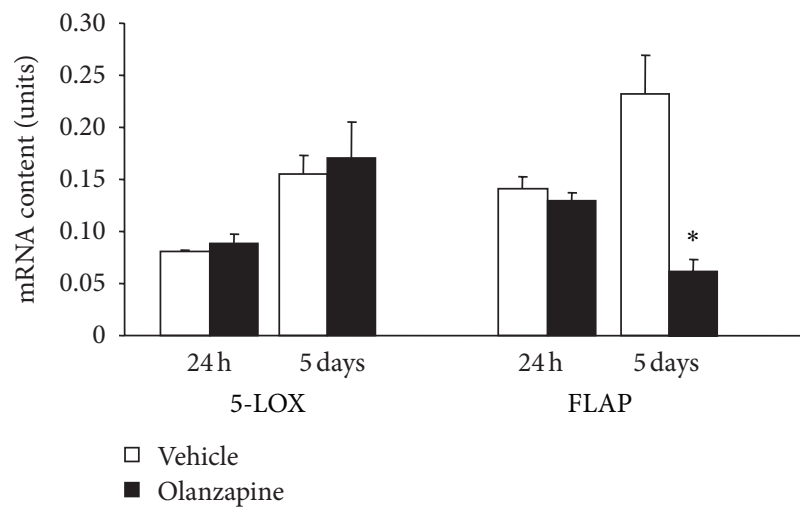

Figure 3: Effect of olanzapine treatment on 5-LOX and FLAP mRNA contents. 3T3-L1 cells were differentiated into adipocytes in the presence and absence of $50 \mu \mathrm{M}$ olanzapine. Samples ( 3 culture dishes per treatment and time point) were collected 24 hours and 5 days after the initiation of the differentiation protocol. The content of 5-LOX and FLAP mRNAs was quantified with a real-time PCR. Data (mean \pm SEM) are expressed as units (coefficient of variation normalized to the corresponding cyclophilin internal control); ${ }^{*} \mathrm{P}<$ 0.05 versus the corresponding vehicle-treated control.

inhibition facilitates the adipogenic effects of olanzapine. Interestingly, we found that exposure of differentiating 3T3L1 cells to MK-886 per se did not cause significant alterations in intracellular lipid content and that this drug altered the adipogenic effects of olanzapine in a concentration-dependent manner. Considering significant differences between the in vitro and in vivo models of drug actions on adipocytes, subsequent in vivo studies with olanzapine and specific FLAP inhibitors are required to evaluate the significance of our in vitro observations.

In conclusion, our results suggest that FLAP-mediated modifications of the 5-LOX system may be considered as a putative mechanism involved in the signaling of lipid dysfunction not only in conditions of inflammation but 


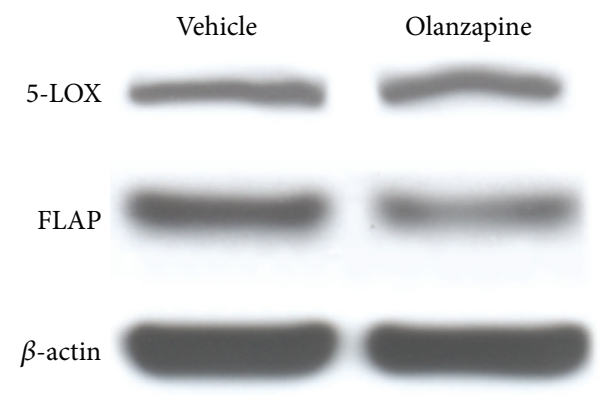

(a)

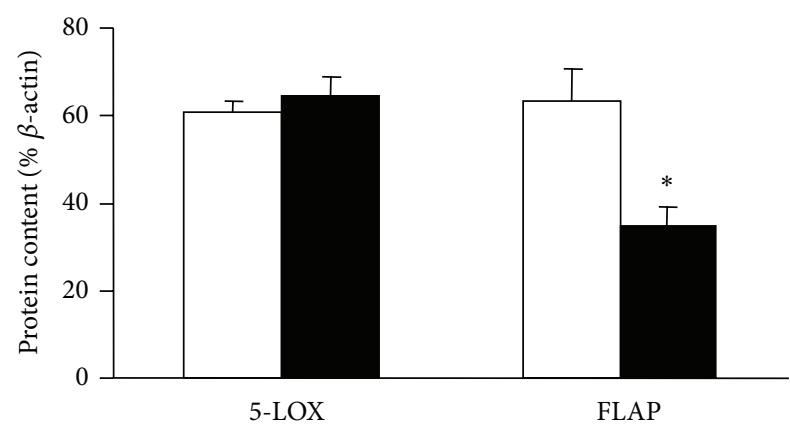

Vehicle

Olanzapine

(b)

FIGURE 4: Effect of olanzapine treatment on 5-LOX and FLAP protein contents. 3T3-L1 cells were differentiated into adipocytes in the presence and absence of $50 \mu \mathrm{M}$ olanzapine. Samples were collected 5 days after the initiation of the differentiation protocol. The content of 5-LOX and FLAP proteins was assayed by quantitative Western blot. The optical density of the 5-LOX and FLAP bands was corrected by the density of the corresponding $\beta$-actin bands (examples shown in a). The results (b) are expressed as a percentage of the corresponding $\beta$-actin values (mean \pm SEM; $n=5$ ); ${ }^{*} P<0.05$ versus the corresponding vehicle-treated control.

possibly in SGAD-related metabolic alterations and adiposity. Furthermore, the observed effects of MK-886 on cellular (e.g., adipocytes) effects of SGADS may involve both FLAPspecific and FLAP-unrelated (e.g., PPAR $\alpha$ ) signaling pathways. We hypothesize that the known polymorphism in the genes of the human FLAP/5-LOX system [16] could play a role in setting a variable individual susceptibility to the peripheral/cellular metabolic side effects of SGADs. This hypothesis could be directly tested in a clinical setting.

\section{Abbreviations}

DMEM: Dulbecco's modified Eagle's medium

FBS: Fetal bovine serum

FLAP: $\quad$ Five lipoxygenase activating protein

IBMX: 3-Isobutyl-1-methylxanthine

5-LOX: 5-Lipoxygenase

SGADs: Second-generation antipsychotic drugs

SREBP-1: Sterol regulatory element binding protein-1.

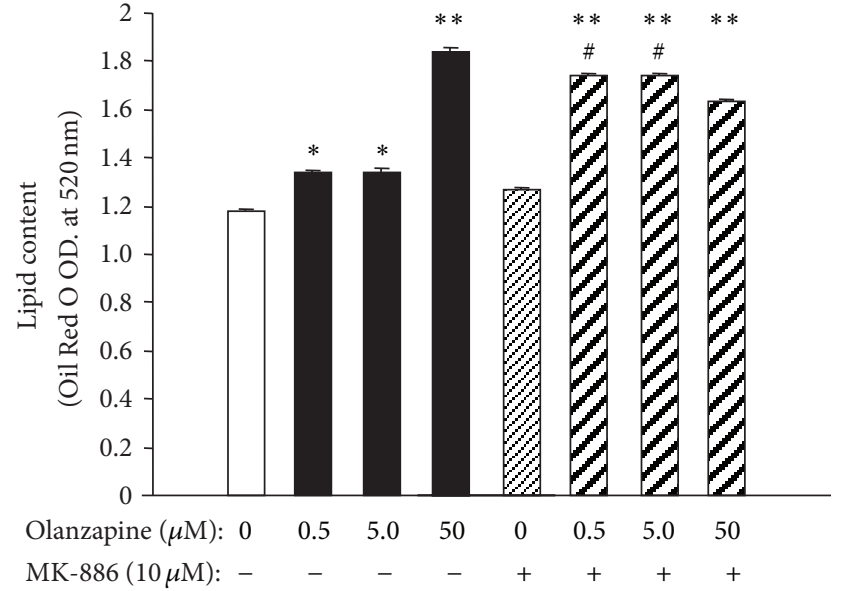

FIGURE 5: Effect of $10 \mu \mathrm{M}$ MK-886 in the presence of increasing concentrations of olanzapine on intracellular lipid content. 3T3-L1 cells were differentiated into adipocytes in the presence and absence of indicated drugs. Samples were collected 5 days after the initiation of the differentiation protocol. Results (mean \pm SEM; $n=4$ ) are expressed as optical density (O.D.) readings at $520 \mathrm{~nm} .{ }^{*} P<0.05$, ${ }^{* *} P<0.001$ versus the corresponding group without olanzapine; ${ }^{\#} P<0.001$ versus the corresponding group without MK-886.

\section{Acknowledgments}

This work was supported by the Psychiatric Institute, University of Illinois at Chicago. The authors thank Radmila Manev, M.D. for helpful discussions.

\section{References}

[1] D. B. Allison, J. W. Newcomer, A. L. Dunn et al., "Obesity among those with mental disorders: a National Institute of Mental Health meeting report," American Journal of Preventive Medicine, vol. 36, no. 4, pp. 341-350, 2009.

[2] R. Coccurello and A. Moles, "Potential mechanisms of atypical antipsychotic-induced metabolic derangement: clues for understanding obesity and novel drug design," Pharmacology o Therapeutics, vol. 127, no. 3, pp. 210-251, 2010.

[3] G. P. Reynolds and S. L. Kirk, "Metabolic side effects of antipsychotic drug treatment-pharmacological mechanisms," Pharmacology \& Therapeutics, vol. 125, no. 1, pp. 169-179, 2010.

[4] T. Baptista, Y. ElFakih, E. Uzcátegui et al., "Pharmacological management of atypical antipsychotic-induced weight gain," CNS Drugs, vol. 22, no. 6, pp. 477-495, 2008.

[5] J. Minet-Ringuet, P. C. Even, M. Goubern, D. Tomé, and R. D. Beaurepaire, "Long term treatment with olanzapine mixed with the food in male rats induces body fat deposition with no increase in body weight and no thermogenic alteration," Appetite, vol. 46, no. 3, pp. 254-262, 2006.

[6] G. D. Cooper, J. A. Harrold, J. C. G. Halford, and A. J. Goudie, "Chronic clozapine treatment in female rats does not induce weight gain or metabolic abnormalities but enhances adiposity: implications for animal models of antipsychoticinduced weight gain," Progress in Neuro-Psychopharmacology \& Biological Psychiatry, vol. 32, no. 2, pp. 428-436, 2008.

[7] V. L. Albaugh, J. G. Judson, P. She et al., "Olanzapine promotes fat accumulation in male rats by decreasing physical activity, 
repartitioning energy and increasing adipose tissue lipogenesis while impairing lipolysis," Molecular Psychiatry, vol. 16, no. 5, pp. 569-581, 2011.

[8] V. L. Albaugh, T. C. Vary, O. Ilkayeva et al., "Atypical antipsychotics rapidly and inappropriately switch peripheral fuel utilization to lipids, impairing metabolic flexibility in rodents," Schizophrenia Bulletin, vol. 38, pp. 153-166, 2012.

[9] J. Minet-Ringuet, P. C. Even, P. Valet et al., "Alterations of lipid metabolism and gene expression in rat adipocytes during chronic olanzapine treatment," Molecular Psychiatry, vol. 12, no. 6, pp. 562-571, 2007.

[10] L. H. Yang, T. M. Chen, S. T. Yu, and Y. H. Chen, "Olanzapine induces SREBP-1-related adipogenesis in 3T3-L1 cells," Pharmacological Research, vol. 56, no. 3, pp. 202-208, 2007.

[11] Z. Yang, J. Y. Yin, Z. C. Gong et al., "Evidence for an effect of clozapine on the regulation of fat-cell derived factors," Clinica Chimica Acta, vol. 408, no. 1-2, pp. 98-104, 2009.

[12] I. Mothe-Satney, C. Filloux, H. Amghar et al., "Adipocytes secrete leukotrienes: contribution to obesity-associated inflammation and insulin resistance in mice," Diabetes, vol. 61, pp. 2311-2319, 2012.

[13] S. K. Chakrabarti, Y. Wen, A. D. Dobrian et al., "Evidence for activation of inflammatory lipoxygenase pathways in visceral adipose tissue of obese Zucker rats," American Journal of Physiology, vol. 300, no. 1, pp. E175-E187, 2011.

[14] R. Horrillo, A. González-Périz, M. Martínez-Clemente et al., "5lipoxygenase activating protein signals adipose tissue inflammation and lipid dysfunction in experimental obesity," The Journal of Immunology, vol. 184, pp. 3978-3987, 2010.

[15] M. Victoriano, R. de Beaurepaire, N. Naour et al., "Olanzapineinduced accumulation of adipose tissue is associated with an inflammatory state," Brain Research, vol. 1350, pp. 167-175, 2010.

[16] K. G. Tantisira and J. M. Drazen, "Genetics and pharmacogenetics of the leukotriene pathway," Journal of Allergy and Clinical Immunology, vol. 124, no. 3, pp. 422-427, 2009.

[17] M. Mehrabian, H. Allayee, J. Stockton et al., "Integrating genotypic and expression data in a segregating mouse population to identify 5-lipoxygenase as a susceptibility gene for obesity and bone traits," Nature Genetics, vol. 37, pp. 1224-1233, 2005.

[18] M. Mehrabian, F. T. Schulthess, M. Nebohacova et al., "Identification of ALOX5 as a gene regulating adiposity and pancreatic function," Diabetologia, vol. 51, no. 6, pp. 978-988, 2008.

[19] M. E. Newcomer and N. C. Gilbert, "Location, location, location: compartmentalization of early events in leukotriene biosynthesis," The Journal of Biological Chemistry, vol. 285, no. 33, pp. 25109-25114, 2010.

[20] M. Kaaman, M. Rydén, T. Axelsson et al., "ALOX5AP expression, but not gene haplotypes, is associated with obesity and insulin resistance," International Journal of Obesity, vol. 30, no. 3, pp. 447-452, 2006.

[21] A. L. Darrow, R. V. Shohet, and J. G. Maresh, "Transcriptional analysis of the endothelial response to diabetes reveals a role for galectin-3," Physiological Genomics, vol. 43, pp. 1144-1152, 2011.

[22] V. Konstantinidou, O. Khymenets, M. I. Covas et al., “Time course of changes in the expression of insulin sensitivity-related genes after an acute load of virgin olive oil," OMICS, vol. 13, no. 5, pp. 431-438, 2009.

[23] S. Dzitoyeva, H. Chen, R. Manev, and H. Manev, "P02-310-a novel mechanism of olanzapine-induced lipid accumulation," European Psychiatry, vol. 26, supplement 1, p. 906, 2011.
[24] S. Dzitoyeva, M. Imbesi, L. W. Ng, and H. Manev, "5Lipoxygenase DNA methylation and mRNA content in the brain and heart of young and old mice," Neural Plasticity, vol. 2009, Article ID 209596, 9 pages, 2009.

[25] O. Rådmark, O. Werz, D. Steinhilber, and B. Samuelsson, "5Lipoxygenase: regulation of expression and enzyme activity," Trends in Biochemical Sciences, vol. 32, no. 7, pp. 332-341, 2007.

[26] O. Firuzi, J. Zhuo, C. M. Chinnici, T. Wisniewski, and D. Praticò, "5-Lipoxygenase gene disruption reduces amyloid- $\beta$ pathology in a mouse model of Alzheimer's disease," FASEB Journal, vol. 22, no. 4, pp. 1169-1178, 2008.

[27] J. Chu and D. Praticò, "The 5-lipoxygenase as a common pathway for pathological brain and vascular aging," Cardiovascular Psychiatry and Neurology, vol. 2009, Article ID 174657, 5 pages, 2009.

[28] J. Chu and D. Praticò, "Involvement of 5-lipoxygenase activating protein in the amyloidotic phenotype of an Alzheimer's disease mouse model," Journal of Neuroinflammation, vol. 9, article 127, 2012.

[29] J. Chu and D. Praticò, "5-Lipoxygenase pharmacological blockade decreases tau phosphorylation in vivo: involvement of the cyclin-dependent kinase-5," Neurobiology of Aging, vol. 34, no. 6, pp. 1549-1554, 2013.

[30] H. Manev, H. Chen, S. Dzitoyeva, and R. Manev, "Cyclooxygenases and 5-lipoxygenase in Alzheimer's disease," Progress in Neuro-Psychopharmacology and Biological Psychiatry, vol. 35, no. 2, pp. 315-319, 2011.

[31] H. Manev and R. Manev, "Benefits of neuropsychiatric phenomics: example of the 5-lipoxygenase-leptin-alzheimer connection," Cardiovascular Psychiatry and Neurology, vol. 2010, Article ID 838164, 2 pages, 2010.

[32] A. González-Périz and J. Clària, "Resolution of adipose tissue inflammation," The Scientific World Journal, vol. 10, pp. 832-856, 2010.

[33] L. Madsen, R. K. Petersen, M. B. Sørensen et al., "Adipocyte differentiation of 3T3-L1 preadipocytes is dependent on lipoxygenase activity during the initial stages of the differentiation process," Biochemical Journal, vol. 375, no. 3, pp. 539-549, 2003.

[34] J. P. Kehrer, S. S. Biswal, E. La et al., "Inhibition of peroxisomeproliferator-activated receptor (PPAR) $\alpha$ by MK886," Biochemical Journal, vol. 356, no. 3, pp. 899-906, 2001. 

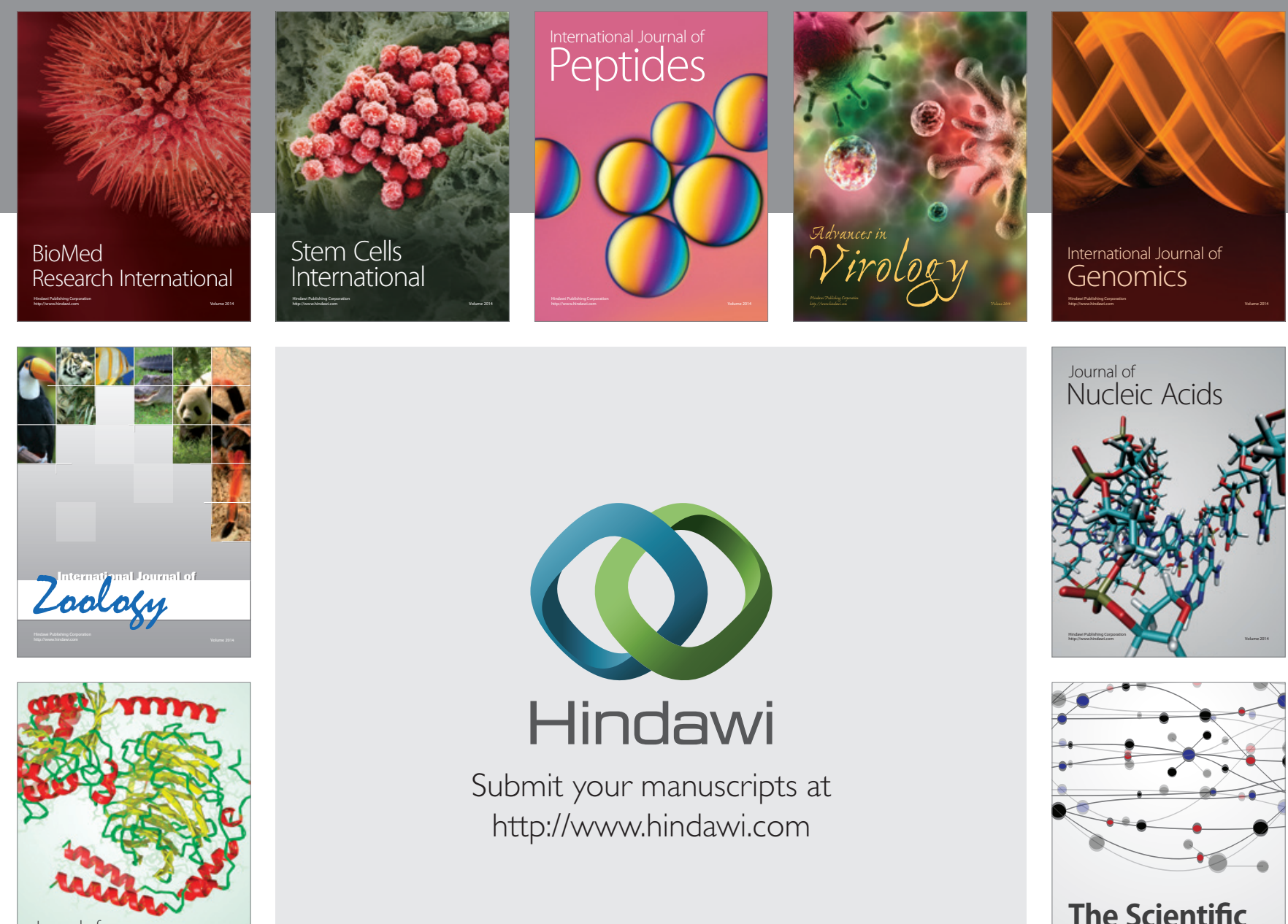

Submit your manuscripts at

http://www.hindawi.com

Journal of
Signal Transduction
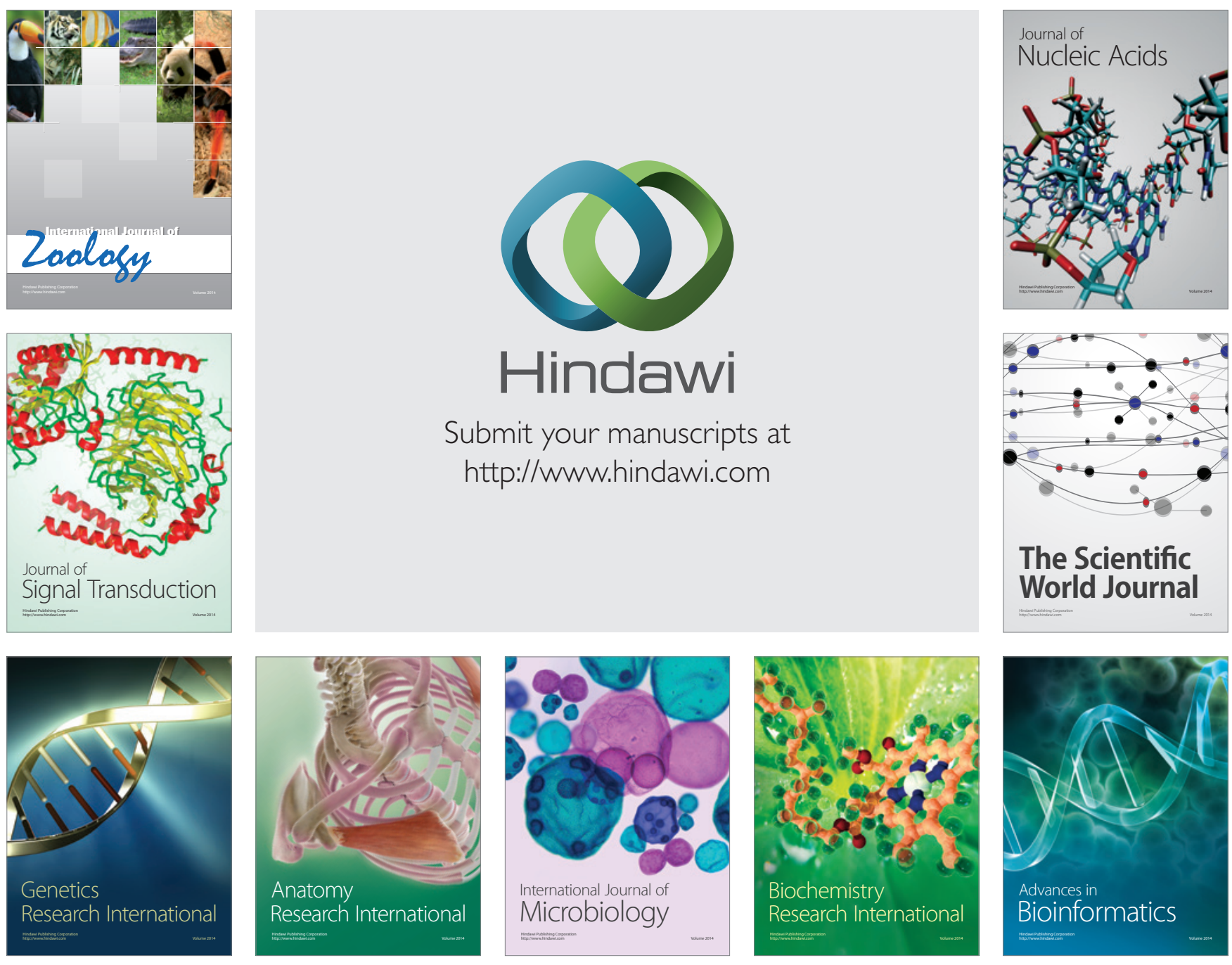

The Scientific World Journal
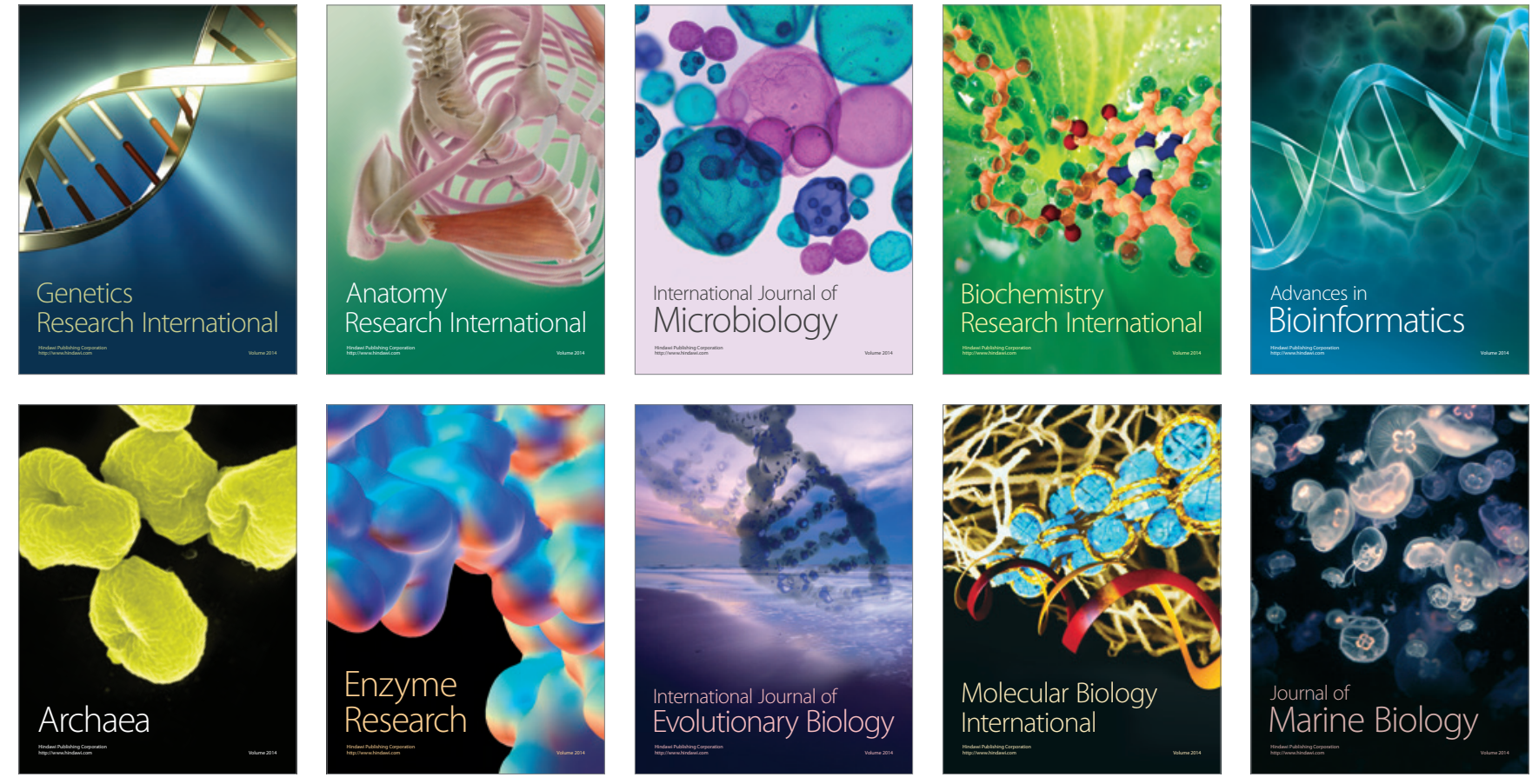